\title{
Electroencephalographic Aspects in Dogs with Congenital Hydrocephalus
}

\author{
Mihaela ARMAȘU ${ }^{1}$, Gabriela Dumitrița STANCIU ${ }^{1}$, Mihai MUSTEAȚA $\breve{~}^{1}$, Diana MOCANU ${ }^{1}$, \\ Gheorghe SOLCAN ${ }^{1}$ \\ ${ }^{1}$ Faculty of Veterinary Medicine, Department of Internal Medicine / Neurology, University of \\ Agricultural Sciences and Veterinary Medicine Ion Ionescu de la Brad Iasi, Romania, 3-8 M. Sadoveanu \\ Alley, 700489 \\ *corresponding author: gsolcan@uaiasi.ro
}

Bulletin UASVM Veterinary Medicine 71(2) / 2014,

Print ISSN 1843-5270; Electronic ISSN 1843-5378

DOI:10.15835/buasvmcn-vm: 10289

\begin{abstract}
Congenital hydrocephalus $(\mathrm{CH})$ is a common neurologic disorder in dogs defined as an increased cerebrospinal fluid volume within the cranial cavity with no any specific causes. The diagnosis of this disease is based on the clinical features and imagistic examination (ultrasonography, CT, MRI) of the brain. Electroencephalography (EEG) is the best electrodiagnostic test in trying to assess the impact of hydrocephalus on electric activity of the brain. The aim of this study is to describe short time EEG changes in dogs with $\mathrm{CH}$.

EEG was performed on 6 dogs with congenital hydrocephalus under general anaesthesia with medetomidine hydrochloride $0.05 \mathrm{mg} / \mathrm{kg}$ inj. EEGs were obtained via five subdermal stainless steel needle electrodes as described by Redding (1978). In EEG recoding the parameters used were: sensitivity $=70 \mu \mathrm{V} / \mathrm{cm}$; time constant $=0.3$ seconds; $\mathrm{Hf}=70 \mathrm{~Hz}$; Lf $=0.5 \mathrm{~Hz}$; notch filter inserted; impedance of all electrodes $<10 \mathrm{k} \Omega$.

The results of the present study revealed abnormalities in EEG background activity in all dogs. Focal abnormalities were seen in 5 patients (slow waves in 2 dogs, spikes and sharp waves in 2 dogs, focal attenuation in 2 cases) and generalized abnormal activities in 4 dogs (generalized slow waves in 4 dogs and generalised spikes and sharp waves in $1 \mathrm{dog})$. The percentage of EEG interictal epileptiform discharges (66.67\% of dogs had spikes, sharp waves, spikes and sharp waves complexes) was greater than seizures clinical seen (33.33\% of dogs).

In conclusion, EEG gived us important information about the severity of changes induced by hydrocephalus. Furthermore, this test is useful to identify seizures associated with hydrocephalus and allows monitoring the therapeutic effects of antiepileptic drugs.
\end{abstract}

Keywords: congenital hydrocephalus, dog, EEG

\section{INTRODUCTION}

Congenital hydrocephalus is a common neurologic disorder in dogs defined as an increased cerebrospinal fluid volume within the cranial cavity, which appears secondary to an excessive production or to an absorption deficit of cerebrospinal fluid (Shihab, 2011; Wünschmann, 2001).

Congenital hydrocephalus is apparent at birth (even if in many cases, clinical signs are first noticed at several months of age) and is caused by stenosis of the mesencephalic aqueduct associated with fusion of the rostral colliculi, genetic factors and in utero exposure to infectious agents and teratogenic chemicals (Thomas, 2010).

Clinical signs of congenital hydrocephalus may include an enlarged, dome-shaped head with persistent fontanelles, ventral or ventro-lateral strabismus due to malformation of the orbitand neurologic deficits including abnormal behavior, cognitive dysfunctions, ataxia, circling, blindness, seizures and vestibular dysfunction. Neurologic deficits may progress over time, remain static, or even improve after 1 to 2 years of age. Affected patients are often fragile and can worsen later in life coincident with other diseases or minor head trauma. 
The diagnosis of this disease is based on the clinical features and imagistic examination (ultrasonography, computer tomography, magnetic resonance imaging) of the brain. Electroencephalography (EEG) is the best electrodiagnostic test in trying to assess the impact of hydrocephalus on electric activity of the brain (Aminoff, 2003). The EEG is a noninvasive investigation, can be reliably repeated without limits and with no adverse effects (Saukkonen, 1988), allows monitoring and the evolution of hydrocephalus in time, and it is not cost-prohibitive as CT or MRI.

Until now, EEG pattern in congenital hydrocephalus was described in children and there are only few studies in dogs. All studies used different protocols to record EEG traces: different number of electrodes, various anesthetics in different doses and different recording time (Pellegrino, 2004; Brauer, 2012; Pákozdy, 2012)

This study aimed to describe short time EEG changes in dogs with congenital hydrocephalus after medetomidine sedation.

\section{MATERIAL AND METHODS}

The study was made on 6 dogs that were presented to Internal Clinic of Faculty of Veterinary Medicine Iasi, which after neurologic and imagistic (CT/MRI) examinations were diagnosed with congenital hydrocephalus (Table 1, Fig. 1)

EEGs were performed with Neurofax $\mathrm{S}$ device (MEB 9400K, Nihon Kohden), after general anaesthesia induced with medetomidine hydrochloride (Domitor, Pfizer), $0.05 \mathrm{mg} / \mathrm{kg}$ inj. i.m. All cases were supervised till complete recovery. No medetomidine antagonist were administered

Tab. 1. Signalments and clinical signs of dogs included in the study

\begin{tabular}{cccccl}
\hline No. & Breed & Sex & Age & Weight $(\mathrm{kg})$ & \multicolumn{1}{c}{ Clinical signs } \\
\hline 1 & Akita Inu & Male & 8 weeks & 2.9 & $\begin{array}{l}\text { Dome shape head, vocalization, dissymmetry, } \\
\text { circling, blindness, ventro-lateral strabismus }\end{array}$ \\
\hline 2 & Chihuahua & Female & 4 years & 3 & Seizures, ataxia \\
\hline 3 & Mixed breed & Male & 8 weeks & 1.5 & $\begin{array}{l}\text { Dome shape head, ventro-lateral } \\
\text { strabismus, blindness, circling }\end{array}$ \\
\hline 4 & Boxer & Female & 3 weeks & 1.5 & Dome shape head, ventro-lateral strabismus \\
\hline 5 & Pug & Male & 10 weeks & 2 & $\begin{array}{l}\text { Dome shape head, dissymmetry, circling, } \\
\text { blindness, ventro-lateral strabismus }\end{array}$ \\
\hline 6 & Yorkshire & Male & 16 weeks & 1.2 & Seizures, vocalisation \\
\hline
\end{tabular}

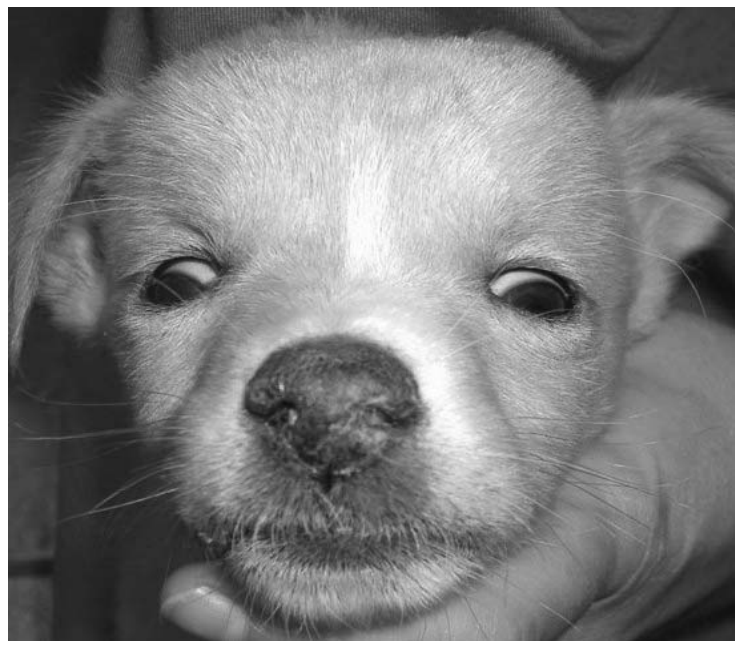

Fig. 1. Two month-old mixed breed dog (case no 3). Congenital hydrocephalus. Ventrolateral strabismus. 


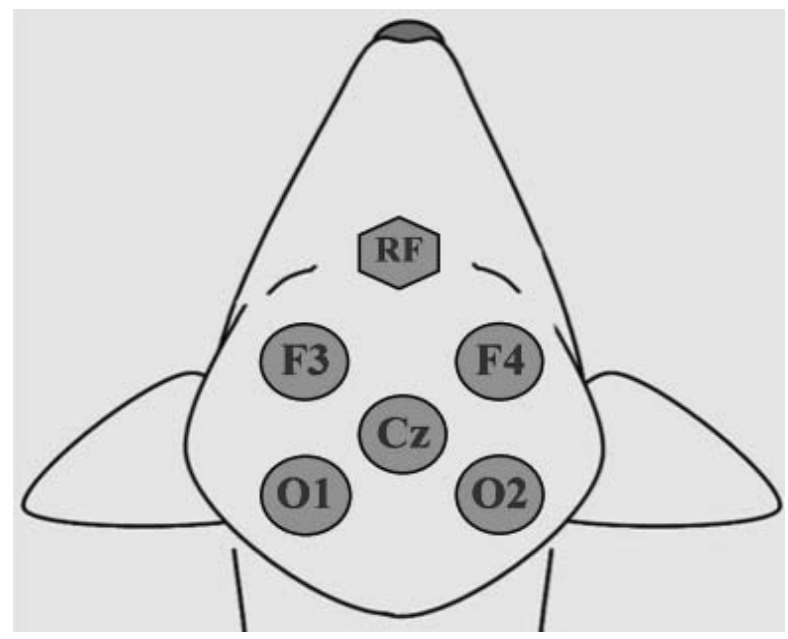

Fig. 2. The electrodes positioned according to Redding pattern (1978):

F3 - frontal left, F4 -frontal right, Cz-vertex,

01 - occipital left, 02 - occipital right, Rf-nose, between the eyes.

as they increased the risk of seizures.EEGs were obtained via five subdermal stainless steel needle electrodes (F3, F4, 01, 02 and $\mathrm{Cz}$ ) as described by Redding (1978) with reference electrode placed on the bridge of the nose (Fig. 2). The area on which the electrodes were placed was trimmed and degreased with alcohol and Skin Pure (Nihon Kohden). In EEG recoding the parameters used were: sensitivity $=70 \mu \mathrm{V} / \mathrm{cm}$; time constant $=$ 0.3 seconds; $\mathrm{Hf}=70 \mathrm{~Hz} ; \mathrm{Lf}=0.5 \mathrm{~Hz}$; notch filter inserted; impedance of all electrodes $<10 \mathrm{k} \Omega$.

Recording sections were visually selected for analysis of background activity using Fast Fourier Transformation (FFT). Spectral bands were, 8.013.0 $\mathrm{Hz}$ for alpha, 13.0-30.0 Hz for beta, 0.5-4.0 $\mathrm{Hz}$ for delta and 4.0-8.0 for theta activity. In order to minimize errors through different skull sizes, forms and thicknesses, the relative power of the spectral bands was calculated for every lead.

By visual examination of the EEGs it was established the EEG pattern (any characteristic EEG activity), background activity (the EEG activity representing the setting in which a given normal or abnormal pattern appears and from which such pattern is distinguish-IFSECN, 1974), all paroxysmal activities (spikes, sharp wave, spikewave complex), as well as possible artefacts. Background activity was categorized into four groups: age-appropriate normal dominant activity, mildly abnormal (dominant activity is greatly restored; slow activity is increased, but subdominant), moderately abnormal (polymor- phic theta-delta waves are dominant, but they are superimposed on a basic rhythm. The mean frequency of the basicrhythm is unchanged, or more or less reduced), significantly abnormal (diffuse polymorphic delta activity with high amplitude and superimposed theta or alpha waves are seen, and the absence of the organized basic rhythm). Generalized slow-wave activity was considered to be present when there were high rhythmic slow waves with constant frequency and shape, which appeared clearly distinct from the basic activity. Focal slow waves were considered to be present when there was a localization of polymorphic theta or delta waves with constant localized amplitude maximum and a steep potential gradient. Focal attenuation was considered to be present when a reduction of $50 \%$ in wave amplitude was observed. Focal spikes and sharp waves were considered focal paroxysmal discharges of spikes or sharp waves with or without slow waves. Generalized spike and sharp-wave activity was categorized under three different forms: diffusely distributed spikes or sharp waves over both hemispheres, or only on one hemisphere, hypsarrhythmia and hypersynchronous spikes or sharp waves over both hemispheres.

\section{RESULTS AND DISCUSSIONS}

In all 6 dogs with congenital hydrocephalus EEG background activity was abnormal (in 2 dogs slightly abnormal, in 3 cases moderately abnormal and in one dog significantly abnormal). Abnormal 
background activity alone was observed in one case. For the other five cases the abnormal background activity was associated with other EEG abnormalities.

Generalized slow waves appeared almost entirely with abnormal dominant activity (4 cases) and only in one case generalized spikes and sharp waves were recorded. Generalized slow wave activity was detected in only one hemisphere for 3 dogs and in 1 dog in both hemispheres. In all cases, the posterior brain areas were free of generalized slow wave activity (table 2).

Focal abnormalities were seen in 5 cases: slow waves were registered in 2 cases, spikes and sharp waves in 2 cases, focal attenuation in 2 cases (table 3).
Spikes and sharp waves activity was observed in 4 cases (Fig. 3, Fig. 4). One of these had generalized spikes and sharp waves seen on both hemispheres and 3 on one hemisphere. One dog had hypsarrhythmia, and another dog had both generalized and focal spikes and sharp waves. Hypersynchronous activity was not observed in any of these patients (table 4).

There are two different etiologies for EEG abnormalities in hydrocephalic dogs. First, the increased intracranial pressure induces disturbances in blood supply - either generally or focally. These EEG abnormalities are the abnormal dominant activity with or without generalized slow-wave paroxysms and focal slow waves, respectively. Another etiology for the EEG

Tab. 2. The prevalence of dominant activity and generalized slow waves and focal slow waves in the electroencephalograms of 6 congenital hydrocephalic dogs

\begin{tabular}{lcc}
\hline & No. of dogs & Percent (\%) \\
\hline DOMINANT BACKGROUND ACTIVITY & & \\
Normal & 0 & 0 \\
Mildly abnormal & 2 & 33.33 \\
Moderately abnormal & 3 & 50 \\
Severely abnormal & 1 & 16.67 \\
\hline GENERALIZED SLOW WAVES & 4 & 66.67 \\
One hemisphere & 3 & 50 \\
Both hemispheres & 1 & 16.67 \\
Posteriorly & 0 & 0 \\
\hline \multicolumn{1}{c}{ FOCAL SLOW WAVES } & 2 & 33.33 \\
\hline
\end{tabular}

Tab. 3. The prevalence of normal/abnormal background activity and others abnormalities in the EEG of 6 congenital hydrocephalic dogs

\begin{tabular}{lcc}
\hline & No. of dogs & Percent (\%) \\
\hline NORMAL EEG & 0 & 0 \\
ABNORMAL DOMINANT ACTIVITY WITH NO OTHERS & 1 & 16.67 \\
ABNORMALITIES & 0 & 0 \\
NORMAL DOMINANT ACTIVITY WITH OTHERS & & \\
ABNORMALITIES & 5 & 83.33 \\
ABNORMAL DOMINANT ACTIVITY WITH OTHERS & & 66.67 \\
ABNORMALITIES & 4 & 33.33 \\
Generalized slow waves & 2 & 33.33 \\
Focal slow waves & 2 & 33.33 \\
Focal attenuation & 2 & 16.67 \\
Focal spikes and sharp waves & 1 &
\end{tabular}




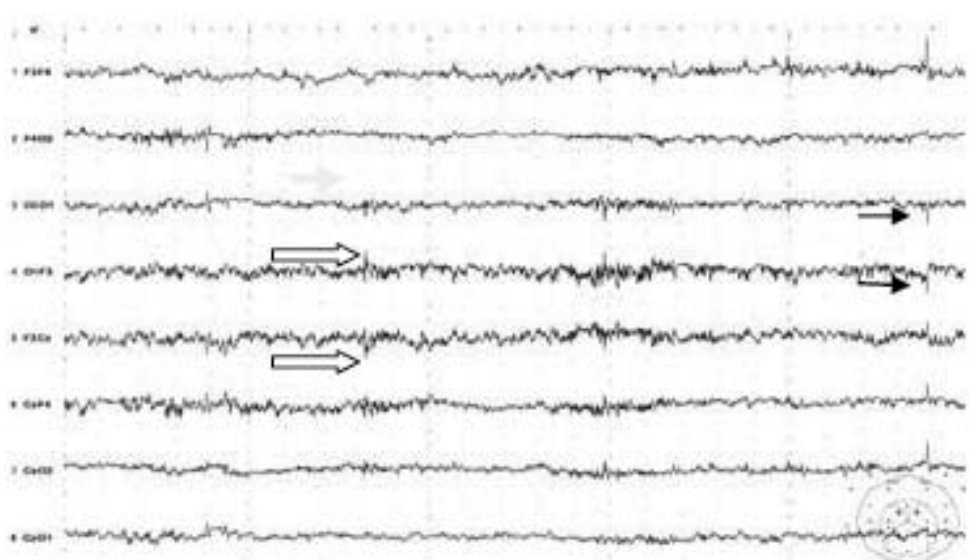

Fig. 3. EEG recording in a Akita Inu dog with congenital hydrocephalus. Spikes (thin arrows) and spikes-sharp waves complexes (thick arrows).

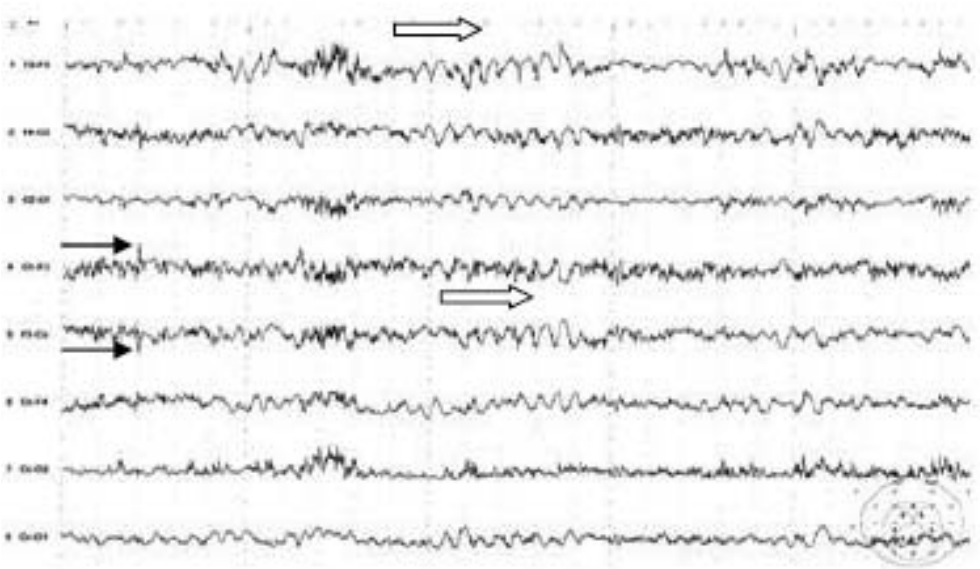

Fig. 4. EEG recording in a mixed breed dog with congenital hydrocephalus. Spikes (thin arrows) and delta waves (thick arrows).

Tab. 4. The prevalence of different types of spikes and sharp waves in the EEG of 6 congenital hydrocephalic dogs

\begin{tabular}{|lcc|}
\hline & No. of dogs & Percentage (\%) \\
\hline SPIKES AND SHARP WAVES & & \\
Focal & 2 & 33.33 \\
Generalized & 1 & 66.67 \\
Hypsarrhythmia & 1 & 16.67 \\
Both & 1 & 16.67 \\
\hline
\end{tabular}

abnormalities in hydrocephalus is represented by the brain lesions due to chronically raised intracranial pressure. In children with hydrocephalus the reported incidence of abnormal EEGs has varied from $20 \%$ to $98 \%$ in different studies (Olischar, 2004). In our study, all dogs had abnormal EEGs. This difference could be due to the fact that in our study EEG was done in dogs with moderate and severe form of hydrocephalus. This hypothesis is confirmed by Saukkonen (1988) who said that when intracranial pressure exceeded 15 $\mathrm{cm} \mathrm{H}_{2} \mathrm{O}$, only $1.9 \%$ of hydrocephalic children $(2$ of 105) had a normal EEG and $6.67 \%$ (7 of 105) had only abnormal dominant activity. Furthermore, Nagarajan (2011) claimed that it is well-known 
that the EEG in babies and infants with moderate to severe hydrocephalus is frequently abnormal.

Generalized abnormalities, abnormal dominant activity and generalized slow wave activity that were observed in all cases, can be interpreted as indicative of brainstem disturbance which, according to Saukkonen (1988), is induced in hydrocephalic patients by cerebrospinal fluid flow blockage.

In 5 cases from this study, hydrocephalus had an acute evolution. Okurowska-Zawada (2007) claimed that a rapid evolution of hydrocephalus may cause substantial brain dysfunctions, which take longer to compensate. When the evolution is chronic, the adaptive mechanisms allow normal functioning of the brain and do not change the bioelectrical activity at the disease onset. This makes that the changes in EEG recording to differ between acute and chronic pathologies and to depend on their intensification, duration or remission. According to Koślacz-Folga (1990), localized EEG changes are most frequently recor-

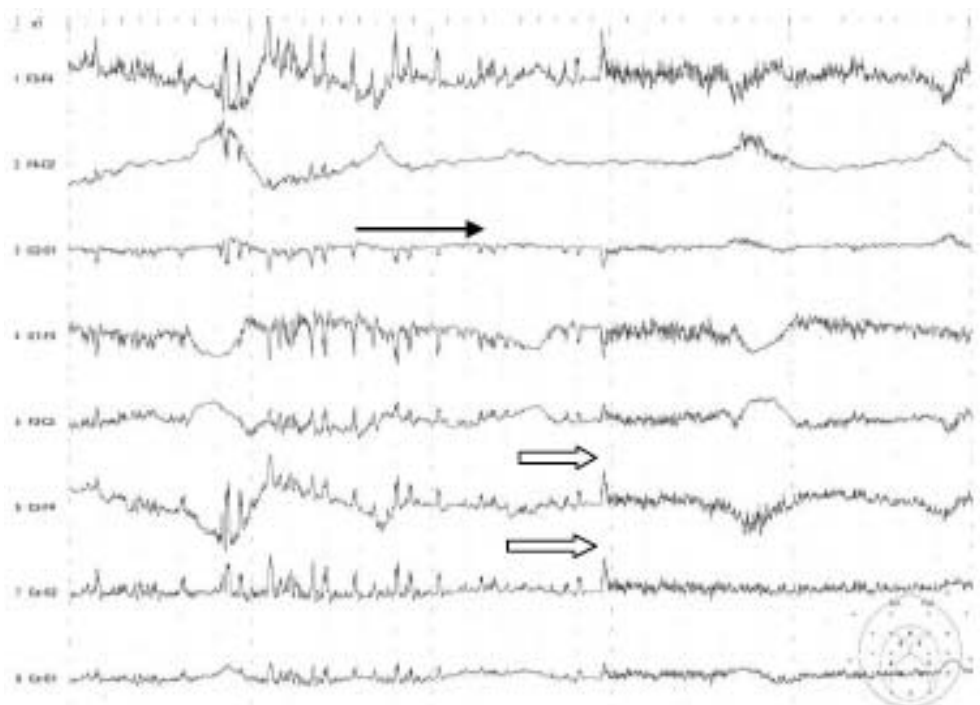

Fig. 4. EEG recording in a 10 weeks Pug dog with congenital hydrocephalus. Spikes and sharp waves activity (thick arrows). Flattened, slower and dysrhythmic background activity (thin arrows).

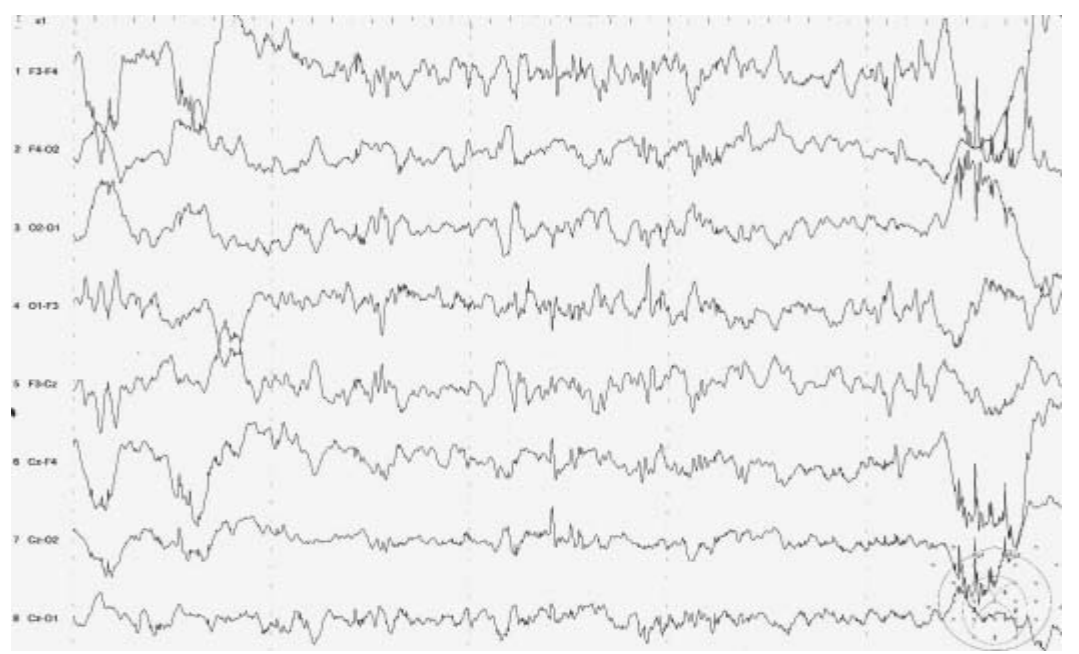

Fig. 5. EEG recording in a 16 weeks Yorkshire terrier with congenital hydrocephalus.

Rare and significant increasing in amplitude EEG wavescharacteristic for hypsarrhythmia (all chanels). 
ded in hydrocephalus. These findings have been observed in our study, focal activity being recorded in all dogs with acute hydrocephalus.

Focal slow activity in the EEG is considered to be induced by metabolic disturbances of adjacent neurons with or without organic brain lesion (Kiloh 1972). In children, slow wave focuses are considered by Saukkonen (1988) to emanate from the cerebral cortex. In this study focal slow activity was detected in 2 cases.

Another EEG aspect presented in 2 hydrocephalic dogs from this study was focal attenuation (Fig.5). This finding may be caused either by focal atrophy or disturbed blood supply due to raised intracranial pressure.

Hypsarrhythmia, a highly disorganized and chaotic brain-wave patterns consisting of high amplitude and irregular waves and spikes was observed in one case (Fig. 6). Haginoya (1999) considered that this type of activity originates from cortical lesions and Burroughs (2014) observed that may be related to cognitive impairment.

In 4 cases with a severe hydrocephalus were noticed high amplitudes and low frequencies. These findings are commonly found in hydrocephalic dogs, but can also be seen in other chronic encephalopathies that affect cortical parenchyma (Harrington 1996; Jaggy 2005). Also, Veggiotti (1998) that observed increased amplitudes in $65 \%$ of hydrocephalic children, put these changes on account of cortical rearrangement in which the dipoles are positioned parallel to one another, and no longer in a radial pattern. According to their study, hydrocephalus that affect the structural organization of the cortex, causing cortical atrophy and thinning, provoke disruption of the organization of the pyramidal cells sufficient to interfere with the genesis of the electrical signal.

The percentage of cases with EEG interictal epileptiform discharges $166.67 \%$ of dogs had spikes, sharp waves, spikes and sharp waves complexes) was higher than those with clinical seizures (33.33\% of dogs). Regarding that, we may assume that EEG abnormalities occur in an early stage (subclinical) of epilepsy. In time, a subclinical epileptic focus is able to become strong enough to determine clinical seizures. Because of that, EEG was very useful in establishing a therapy in order to keep quiet those brain areas responsible by the genesis of the epileptic discharges.
Acknowledgment: This paper was published under the frame of European Social Fund, Human Resources Development Operational Programme 2007-2013, projectno. POSDRU/159/1.5/S/132765.

\section{CONCLUSIONS}

EEG is may be an useful exam in the assessment of brain bioelectrical activity since it allows to detect subclinical epileptic discharges.

We conclude that in order to establish the prognosis, and to give a more specific treatment, EEG must be a valuable exam in the assessment of the hydrocephalic patient.

\section{REFERENCES}

1. Aminoff M. J., 2003 - Electrophysiology, In: Goetz C.G. - Textbook of Clinical Neurology, $2^{\text {nd }}$ Ed., Saunders, Philadelphia.

2. Brauer C., Kästner B.R.S., Rohn K., Schenk C.H., Tünsmeyer J., Tipold A., 2012 - Electroencephalographic recordings in dogs suffering from idiopathic and symptomatic epilepsy: Diagnostic value of interictal short time EEG protocols supplemented by two activation techniques. The Veterinary Journal 193, 185-192.

3. Burroughs S.A., Morse R.P., Mott S.H., Holmes G.L., 2014. Brain connectivity in West syndrome. Seizure. 2: S10591311(14)00098-3.

4. Haginoya K., Kon K., Tanaka S., Munakata M., Kato R., Nagai M., Yokoyama H., Maruoka S., Yamazaki T., Iinuma K., 1999. The origin of hypsarrhythmia and tonic spasms in West syndrome: evidence from a case of porencephaly and hydrocephalus with focal hypsarrhythmia. Brain and Development, 21(2), 129-131.

5. Harrington M.L., Bagley R.S., Moore M.P., 1996. Hydrocephalus. The Veterinary Clinics of North America (Small Animal Practice) Intracranial Disease 26, 843-56.

6. Jaggy A., 2005. Atlas und Lehrbuch der Kleintierneurologie. 1st edn. Hannover: Schlütersche.

7. Kiloh L.G., McComas A.J., Osselton J.W., 1972. Clinical electroencephalography, 3rd edn. Butterworth, London.

8. Koślacz-Folga A. Elektroencefalografia wieku rozwojowego. Warszawa: PZWL; 1980.

9. Okurowska-Zawada B., Sobaniec W., Kułak W., ŚmigielskaKuzia J., Paszko-Patej G., Sienkiewicz D., Sendrowski K., 2007 Clinical-electroencephalographic analysis of brain bioelectrical activity in children with myelomeningocele and internal hydrocephalus. Advances in Medical Sciences, Vol. 52 (1) 200-3.

10. Olischar M., Klebermass K., Kuhle S., Hulek M., Messerschmidt A., Weninger M., 2004. Progressive posthemorrhagic hydrocephalus leads to changes of amplitude integrated EEG activity in preterminfants. Childs Nerv Syst 20:41-45.

11. Pákozdy Á., 2012 - Important Aspects of Canine Idiopathic and Symptomatic Epilepsy. Doctoral thesis, Szent István University and University of Veterinary Medicine Vienna. 
12. Pellegrino C.F., Sica E.P.R., 2004 - Canine electroencephalographic recording technique: findings in normal and epileptic dogs. Clinical Neurophysiology 115, 477-487.

13. Saukkonen A.L., 1988. Electroencephalographic findings in hydrocephalic prior to initial shunting. Child's Nerv Syst. 4:339-343.

14. Shihab N., Davies E., Kenny P.J., Loderstedt S. and Volk H.A. 2011, Treatment of Hydrocephalus with Ventriculoperitoneal Shunting in Twelve Dogs. Veterinary Surgery, 40: 477-484.
15. Thomas W.B., 2010. Hydrocephalus in Dogs and Cats. Vet Clin Small Anim., 40, 143-159.

16. Veggiotti P, Beccaria F, Papalia G, Termine C, Piazza F, Lanzi G., 1998. Continuous spikes and waves during sleep in children with shunted hydrocephalus. Childs Nerv Syst:;14(4-5):188-94.

17. Wünschmann A, Oglesbee M., 2001, Periventricular changes associated with spontaneous canine hydrocephalus. Vet Pathol.;38:67-73 